Reprod. Nutr. Dévelop., 1985, 25 (4 A), 605-618.

\title{
Relations mère-jeune et réponse prolactinique à la stimulation mammaire chez la vache : Influences de la traite et de l'allaitement libre ou entravé
}

\author{
O. PEREZ, Nelly JIMENEZ de PEREZ, P. POINDRON, P. LE NEINDRE $\left({ }^{*}\right)$, \\ J. P. RAVAULT
}

Station de Physiologie de la Reproduction, I.N.R.A., Nouzilly, 37380 Monnaie, France.

(*) Laboratoire de Production de Viande, I.N.R.A., Theix, 63122 Ceyrat, France.

Summary. Influence of management conditions after calving on mother-young relationships and PRL response to mammary stimulation in the cow.

Maternal behaviour and PRL response to mammary stimulation have been studied in three groups of 20 Friesian cows each, held according to three different systems of management. In the 1st group (group AL), the cows suckled 3 alien calves and were all kept in one pen after a fostering period of two weeks. In the second group (group $A E$ ), the cows suckled 3 alien calves twice a day when tethered. In this group, the fostering procedure was limited to $18 \mathrm{~h}$. In the 3rd group (group T), the cows were separated from their calves just after birth and then milked twice a day.

On day 45 after calving, mother-young relationships were observed in the three groups for one day, the animals all being kept with familiar or alien calves on this occasion. Then, on day 112 postpartum, the PRL response to suckling (groups $A L$ and $A E$ ) or to milking (group T) was studied in 4 cows of each group.

Group AL cows were maternal and selective. They preferentially suckled their foster calves rather than alien ones and suckling was carried out in parallel-inverse position. Group $A E$ cows were also maternal but the relationships were not selective and suckling in parallel-inverse position was less frequent. Finally, most milked cows did not allow the calves to suck.

A comparison of PRL response to mammary stimulation showed that the response of milked cows (group T) to milking was low whereas suckling by an alien calf in group AL did not lead to a PRL response ; the presence of the 3 foster calves was needed to induce this response. These results indicate that breeding conditions after calving influence motheryoung relationships and suggest that maternal behaviour is a modulating factor of PRL response to suckling.

\section{Introduction.}

La libération de la prolactine (PRL) lors de la tétée ou de la traite est un phénomène bien établi chez les Mammifères domestiques. C'est le cas chez la brebis (Fell et al., 1972 ; Kann et al., 1978), la chèvre (Bryant et al., 1968 ; Johke, 1970 ; 
Hart, 1974) et la vache (Johke, 1969 ; Fell, 1971 ; Tindal, 1974). Parmi les facteurs influençant la libération de cette hormone chez la vache, la durée et l'intensité de la stimulation mammaire sont sans doute ceux qui ont été les mieux analysés (Tucker, 1971 ; Karg et Schams, 1974 ; Reinhardt et Schams, 1975 ; Gordon et al., 1979). Par contre, le rôle joué par d'autres facteurs, dont ceux associés à l'existence d'une motivation maternelle, restent peu étudiés dans cette espèce. Des travaux réalisés chez la rate indiquent cependant que les stimulations tactiles ne sont pas les seules informations sensorielles susceptibles d'influencer la sécrétion de prolactine au moment de l'allaitement. Dans cette espèce, la seule présence des ratons induit une décharge de prolactine très marquée (Grosvenor et Mena, 1971). II semble donc que l'existence du lien mère-jeune peut influencer de façon non négligeable la décharge de prolactine chez la femelle allaitante.

Chez la vache, le comportement maternel se caractérise par la mise en place d'une relation sélective entre la mère et son veau, au cours d'une période privilégiée située juste après la parturition (Hudson et Mullord, 1977 ; Le Neindre et Garel, 1976). Dans la mesure où une relation mère-jeune bien caractérisée existe chez la vache, il est donc possible que, comme chez la rate, la décharge de prolactine lors de la tétée soit liée en partie à l'existence des relations mère-jeune. Afin de vérifier cette hypothèse, nous avons cherché à savoir : (1) en quoi le mode de conduite après le vêlage (traite ou allaitement) pouvait influencer le comportement maternel ; (2) s'il existait une relation entre l'existence d'un comportement maternel sélectif et la décharge de PRL chez la vache.

L'influence de ces différents modes de conduite sur la production laitière de ces vaches a déjà fait l'objet d'une publication (Perez et al., 1983).

\section{Matériel et méthodes.}

A. - Animaux et mode de conduite après le vêlage.

Soixante vaches multipares de race Frisonne Pie Noire ont été réparties en 3 lots identiques de 20 animaux. L'alimentation a été identique pour les 3 lots. Tous les animaux utilisés dans cette expérience ont vêlé au cours d'une période de 31 jours, les $3 / 4$ des vêlages ayant eu lieu pendant le mois de septembre.

Les 3 lots expérimentaux correspondaient à 3 modes de conduite à partir du vêlage : - vaches allaitantes libres (lot $A L$ ) ; - vaches allaitantes entravées (lot AS) : - vaches traites (lot $\mathrm{T}$ ).

LOT AL : Vaches allaitantes « libres ». - Dès le vêlage, chaque vache était séparée de son veau en évitant tout contact entre les deux animaux. Le plus rapidement possible après le vêlage (66 $\pm 59 \mathrm{~min}$ ), chaque nourrice était enfermée dans un box de $16 \mathrm{~m}^{2}$ environ, avec 3 veaux étrangers de même âge jusqu'à acceptation des veaux à la tétée sans intervention humaine len moyenne 15,5 $\pm 2,7$ jours). Nous appellerons ces veaux "légitimes ", par opposition aux autres veaux du troupeau, que nous nommerons veaux " étrangers ». 
Après cette période, et jusqu'au sevrage des veaux (mois de mai-juin) à l'âge de 8 mois environ, les animaux ont été rassemblés par groupe de 10 vaches et 30 veaux dans une stabulation libre, avec une aire commune aux vaches et aux veaux de $170 \mathrm{~m}^{2}$ et une zone de $92 \mathrm{~m}^{2}$ réservée aux veaux.

Lot $A E$ : Vaches allaitantes "entravées". - Peu de temps après le vêlage $(69 \pm 55 \mathrm{~min})$, chaque vache a passé une période de temps réduite (18 $\pm 8 \mathrm{~h})$ dans un box avec 3 veaux étrangers. Cette période n'avait pas pour but d'assurer des adoptions, mais simplement de permettre à la vache d'établir un comportement d'allaitement sans que celui-ci soit sélectif. Après cette période, les vaches et les veaux étaient séparés, sauf lors des tétées journalières qui avaient lieu sous surveillance vers 6 et $16 \mathrm{~h}$. Du fait de l'espace limité, ces tétées surveillées avaient lieu en deux temps; les 5 premières vaches et les 15 premiers veaux sortant de leurs parcs formaient un premier groupe; les animaux restants en formaient un second. Les vaches étaient alors attachées au cornadis et les veaux pouvaient choisir les vaches sous lesquelles ils allaient téter. La possibilité pour les veaux de changer de nourrice à chaque période d'allaitement avait pour but de diminuer les chances d'établissement tardif d'un comportement sélectif de la part des mères. Dans ce groupe il n'existe donc pas de veaux "légitimes " ou "étrangers ", comme cela est d'ailleurs confirmé par les résultats. Une personne surveillait les tétées matin et soir pour que chaque vache allaite effectivement 3 veaux. Afin d'assurer une alimentation suffisante à chacun, ceux-ci ne changeaient pas de nourrice au cours de la tétée. Un égouttage manuel a été dans quelques cas nécessaire au cours des premiers jours de la lactation, à cause d'une production laitière supérieure au besoin des veaux.

Lot $T$ : Vaches traites. - Elles n'ont jamais eu de contact avec des veaux sauf pendant la période d'observation des relations mère-jeune et étaient traites à la machine 2 fois par jour vers 6 et $16 \mathrm{~h}$, dans une salle de traite en épi de marque Alfa-Laval de type Duovac. Les principales caractéristiques de la machine à traire étaient les suivantes : - Niveau de vide : $42 \mathrm{kPa}$; - Lactoduc ligne basse ; - Vitesse de pulsation : $55 \mathrm{p} / \mathrm{min}$.

Il n'y avait ni préparation de la mamelle à la traite ni égouttage, mais le système Duovac permet une dépression faible au début et à la fin de la traite.

\section{B. - Mesures.}

1. Observations des relations mère-jeune en lot: Les observations comportementales ont été réalisées aux environs de 45 jours de lactation dans les trois lots expérimentaux. Les tentatives de tétée réussies ou non, les allaitements, ainsi que leur durée et leur position ont été notés.

Pour toutes les allaitantes, les vaches et les veaux étaient en liberté dans leur case habituelle pendant 2 jours consécutifs de l'aube au crépuscule. Pour les allaitantes surveillées, vaches et veaux ont été rassemblés la veille des observations après la tétée du soir. Pour faciliter la manipulation des animaux ainsi que leur observation, chacun des groupes expérimentaux $A L$ et $A E$ de 20 vaches a été divisé en 2 sous-groupes (10 vaches et 30 veaux). 
Pour les vaches soumises à la traite, un groupe a été observé lors de la mise en contact avec 20 veaux provenant du lot des allaitantes surveillées et l'autre lors de la mise en contact avec 20 veaux provenant des allaitantes libres ( 1 veau par vache a été laissé avec les nourrices pour que celles-ci soient tétées). Dans les deux cas, vaches et veaux ont été rassemblés dans le parc la veille des observations après la traite du soir. La durée des observations pour ces 2 groupes de vaches traites a été limitée à une journée car certaines vaches n'ont pas du tout allaité pendant la journée d'observation et il était donc indispensable de les traire.

Les animaux étaient identifiés par des chiffres et/ou des lettres peints de chaque côté du corps. Toutes les relations vache-veau ont été enregistrées sur un magnétophone portatif, l'observateur étant placé dans une cabine située à $2,5 \mathrm{~m}$ environ du sol, sur le mur d'un des côtés du parc. Cette technique a permis d'observer les animaux sans apparemment les perturber.

Les relations mère-jeune ont été caractérisées par les tétées et les léchages : nous avons considéré qu'un veau tétait effectivement lorsqu'il avait le trayon dans la bouche et qu'il le conservait pendant une période minimale de $2 \mathrm{~min}$. Lorsque ce temps était inférieur, nous avons considéré qu'il y avait eu une " tentative de tétée non réussie " due soit à la nourrice qui refusait le jeune, soit au veau qui abandonnait le trayon avant $2 \mathrm{~min}$.

Nous avons distingué 4 positions de tétée : a) position parallèle inversée (A0) par rapport au corps de la nourrice ; c'est la position prise le plus fréquemment par les Ongulés (Fraser, 1968 ; Poindron et Le Neindre, 1975) ; b) perpendiculaire (A1) par rapport à la nourrice ; c) arrière (A2) entre les pattes arrières de la nourrice ; d) double ou triple (A3) lorsque le veau tète à côté d'un ou plusieurs autres déjà placés en $A 0$ ou $A 1$.

Lors des enregistrements, nous avons noté l'heure du début et de la fin de chaque événement. Ces données ont permis de calculer, à une minute près, la durée de chaque tétée (intervalle entre le moment où le veau prend le trayon, et le moment où il le laisse définitivement) ; quand les interruptions ont été inférieures à 5 min sans changer de nourrice, nous avons considéré que le veau avait tété une seule fois.

Pour compléter notre information sur la tétée, nous avons enregistré également l'ordre d'arrivée des veaux sous chaque nourrice et l'identité de la vache sous laquelle ils avaient tété.

2. Etude de la réponse prolactinique à la tétée ou à la traite : Afin de préciser l'influence de la relation mère-jeune sur la décharge de prolactine lors de la stimulation mammaire, des prises de sang ont été effectuées sur quatre animaux de chacun des 3 lots expérimentaux, à 112 jours post-partum. Les mesures n'ont pu être effectuées plus tôt à cause d'autres impératifs associés au protocole expérimental (étude des retours en chaleur notamment). Les animaux ont été choisis en fonction des observations comportementales :

Lot $A L: 4$ vaches maternelles et sélectives (c'est-à-dire n'allaitant que leurs veaux légitimes et refusant les étrangers).

Lot $A E$ : 4 vaches maternelles et non sélectives (laissant téter n'importe quel veaul. 
Lot $T: 4$ vaches traites (non maternelles refusant d'allaiter les veaux).

Séquences des prises de sang. - Les prélèvements étaient réalisés à I'aide de cathéters implantés dans la jugulaire la veille du début des prélèvements. Les animaux étaient habitués à la manipulation par les expérimentateurs et n'ont montré aucun signe de perturbation dû aux prélèvements. Au cours de 3 jours consécutifs, les prises de sang ont débuté à $16 \mathrm{~h}$ et ont été répétées toutes les $5 \mathrm{~min}$ pendant 95 min (avant, pendant et après la stimulation mammaire). La stimulation mammaire (tétée pour les groupes $A L$ et $A E$, traite pour le groupe $T$ ) a eu lieu $36 \pm 11$ min après le début des prélèvements.

Les prélèvements ont été effectués de la façon suivante :

Lot $A L$ : Le premier jour, l'évolution du taux plasmatique de PRL était étudiée pendant la tétée d'un des 3 veaux adoptés par la vache. Le deuxième jour, la vache était soumise à la tétée d'un veau étranger, et le $3^{e}$ jour, les 3 veaux adoptés tétaient en même temps sous leur nourrice. Lors des prélèvements, les animaux étaient en liberté dans une case de $4 \mathrm{~m} \times 4 \mathrm{~m}$.

Lot $A E$ : Les prélèvements étaient réalisés 3 jours consécutifs lors de la tétée de chaque nourrice par 3 veaux du lot (choisis au hasard) dans les mêmes conditions que lors des tétées journalières au cornadis et sous surveillance.

Lot $T$ : Les prises de sang étaient faites 3 jours de suite lors de la traite mécanique.

Dosage radioimmunologique. - Les prélèvements ont été immédiatement centrifugés et le plasma obtenu stocké à $-15^{\circ} \mathrm{C}$ jusqu'au moment du dosage. Celui-ci a été réalisé selon la technique radioimmunologique de double anticorps spécifique décrite par Kann (1971) pour la PRL ovine. Les dilutions de plasma bovin donnent des résultats similaires à ceux obtenus avec le standard ovin. Les résultats sont exprimés en ng NIH-PS6 par $\mathrm{ml}$ de plasma.

\section{Résultats.}

\section{A - Relations mère-jeune en fonction du mode de conduite.}

Les relations entre les vaches et les veaux sont très différentes selon les lots.

La fréquence des tétées et les temps totaux d'allaitement sont plus élevés dans le lot $A E$ que dans les deux autres lots. Par contre la durée moyenne de chaque allaitement est plus longue chez les allaitantes libres que chez les autres vaches (tabl. 1). Cependant, ces données moyennes par lot ne rendent pas compte de la plus faible variabilité des différents critères (nombre d'allaitements, temps d'allaitement, durée des allaitements) pour les animaux du lot AL que pour ceux des deux autres lots. En particulier, dans le lot T, 3 vaches n'ont pas été tétées de toute la journée alors que 3 autres l'étaient très fréquemment. De la même façon le nombre de tétées par veau est différent selon les lots. Les veaux du lot $A E$ tètent plus souvent que ceux du lot $A L$ et surtout que ceux du lot $T$. Le nombre de veaux tétant en moyenne par allaitement est très différent suivant les lots (AL : $2,6 ; \mathrm{AE}: 1,9 ; \mathrm{T}: 1,0)$. 
Influence du mode de conduite des vaches sur les relations mère-jeune lors de la tétée (moyenne \pm écart-type) - données ramenées à une journée d'observation

\begin{tabular}{|c|c|c|c|}
\hline & $\begin{array}{l}\text { Allaitantes } \\
\text { libres }(A L)\end{array}$ & $\begin{array}{c}\text { Allaitantes } \\
\text { entravées (AE) }\end{array}$ & Traites (T) \\
\hline $\begin{array}{l}\text { Allaitement par vache : } \\
\text { Nombre/jour }\end{array}$ & $\begin{array}{r}4,25 a \\
+\quad 1,92 a\end{array}$ & $\begin{array}{r}8,02 \mathrm{~b} \\
+\quad 3,67 \mathrm{~b}\end{array}$ & $\begin{array}{r}3,6 \mathrm{a} \\
\pm \quad 4,76 \mathrm{~b}\end{array}$ \\
\hline Temps total (min) & $\begin{array}{r}50,4 \text { a } \\
+19,64 \text { a }\end{array}$ & $\begin{array}{r}72,3 \mathrm{~b} \\
\pm 34,9 \mathrm{~b}\end{array}$ & $\begin{array}{r}38,0 a \\
\pm 49,55 \mathrm{~b}\end{array}$ \\
\hline Durée/allaitement (min) & $\begin{array}{r}12,22 \mathrm{a} \\
\pm \quad 2,18 \mathrm{a}\end{array}$ & $\begin{array}{r}9,07 \mathrm{~b} \\
+\quad 1,84 \mathrm{a}\end{array}$ & $\begin{array}{r}8,13 \mathrm{~b} \\
\pm \quad 5,48 \mathrm{~b}\end{array}$ \\
\hline $\begin{array}{l}\text { Tétée par veau : } \\
\text { Nombre total }\end{array}$ & $\begin{array}{r}3,66 \mathrm{a} \\
\pm \quad 1,48 \mathrm{a}\end{array}$ & $\begin{array}{r}5,01 \mathrm{~b} \\
\pm \quad 1,79 \mathrm{a}\end{array}$ & $\begin{array}{r}1,77 \mathrm{c} \\
\pm \quad 0,88 \mathrm{~b}\end{array}$ \\
\hline Temps total $(\mathrm{min})$ & $\begin{array}{r}29,3 \text { a } \\
+11,1 \text { a }\end{array}$ & $\begin{array}{r}34,5 \mathrm{~b} \\
+13,6 \mathrm{a}\end{array}$ & $\begin{array}{r}13,9 c \\
\pm \quad 7,2 \mathrm{~b}\end{array}$ \\
\hline Durée/tétée (min) & $\begin{array}{rl}7,8 & a b \\
\pm \quad 2,7 & a\end{array}$ & $\begin{array}{r}7,0 \mathrm{a} \\
+\quad 2,0 \mathrm{a}\end{array}$ & $\begin{array}{r}8,4 \mathrm{~b} \\
\pm \quad 4,1 \mathrm{~b}\end{array}$ \\
\hline
\end{tabular}

Les moyennes ou les écarts-types ayant des lettres non identiques sont statistiquement différents $(P<0,05)$.

Aucune différence dans les paramètres d'allaitement n'est observée entre les 2 groupes de veaux provenant des lots $A L$ et $A E$ lors de leur mise en présence avec les vaches habituellement traites. Enfin, chez les allaitantes entravées aucune différence $n$ 'est observée dans les relations existant entre les nourrices et les veaux avec lesquels elles avaient été confinées pendant $18 \mathrm{~h}$ après le vêlage d'une part et les relations existant entre ces mêmes nourrices et les autres veaux du lot d'autre part.

Les veaux légitimes des vaches allaitantes libres tètent beaucoup plus fréquemment en position parallèle-inversée que les veaux étrangers ou que ceux des autres lots et la réussite des essais est plus fréquente (tabl. 2). Les vaches du lot T provoquent dans $55 \%$ des cas l'insuccès des tétées alors que cette proportion n'est que de $11 \%$ pour celles du lot $A E$ et de $20 \%$ pour celles du lot $A L$.

En ce qui concerne les léchages, les vaches du lot $A E$ ont beaucoup plus léché les veaux que celles des autres lots et les vaches du lot $A L$ lèchent beaucoup plus fréquemment les veaux légitimes que les étrangers.

Enfin, dans le lot des allaitantes libres il n'est pas possible de faire une distinction nette entre les vaches ayant bien adopté leur veau et celles pour lesquelles le lien est plus lâche. En effet, comme le montre la fig. 1, le rapport du temps d'allaitement sous la nourrice légitime et sous des vaches étrangères diminue progressivement et il n'existe pas de séparation précise lorsqu'on passe des veaux tétant principalement la mère adoptive à ceux tétant de préférence des autres nourrices. 


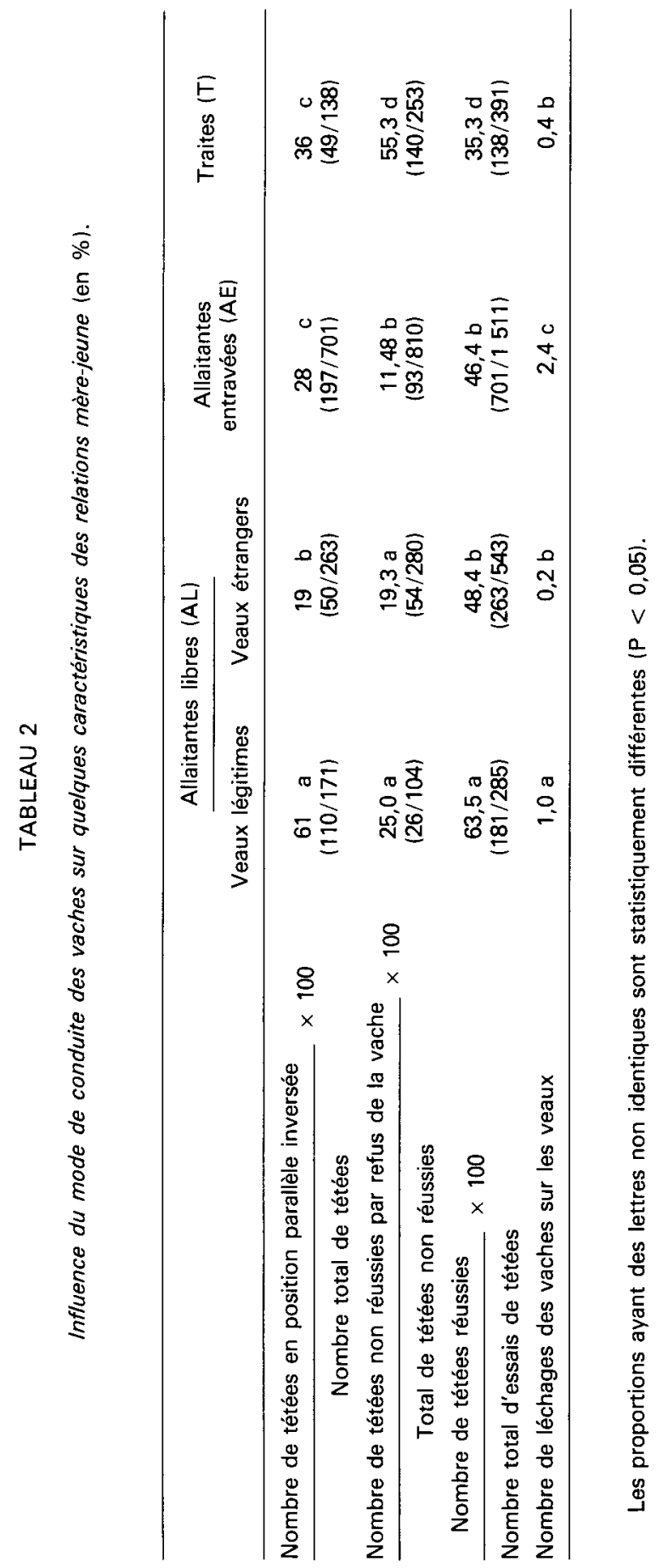




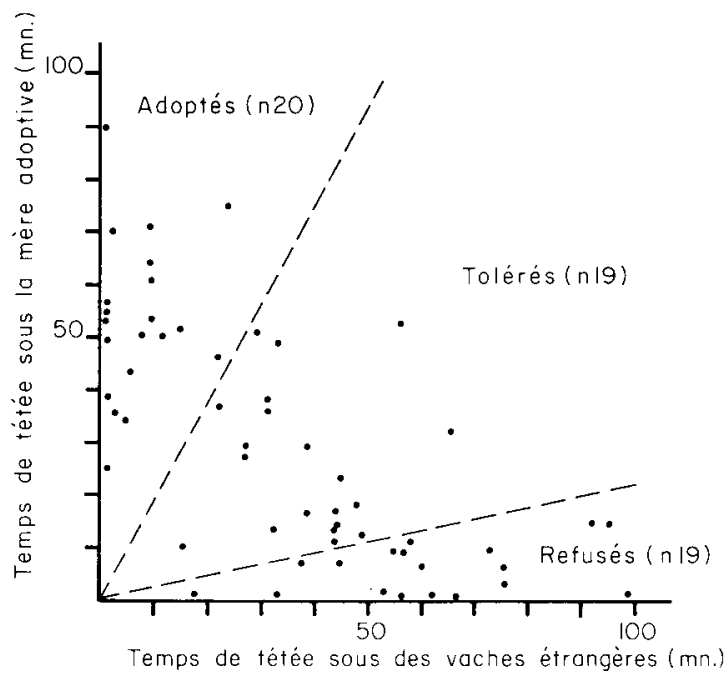

FIG. 1. - Distribution des veaux selon leur comportement à la tétée.

\section{$\mathrm{B}$ - Etude de la réponse prolactinique à la stimulation mammaire.}

Les différentes réponses prolactiniques pour les 3 lots expérimentaux sont représentées dans les fig. 2,3 et 4 ; elles sont différentes selon le mode de conduite d'une part (allaitement libre, allaitement entravé et traite 2 fois par jour) et selon l'identité du veau allaité (légitime ou étranger).

Les concentrations moyennes de prolactine sont calculées en faisant la moyenne des valeurs obtenues dans chaque groupe pour une situation donnée avant le début de la stimulation mammaire par le veau ou la machine à traire, pendant la stimulation, et après l'arrêt de celle-ci (cf. fig. 2, 3, 4). La réponse prolactinique $\mathrm{R}$ est égale à la différence des concentrations moyennes ainsi obtenues avant et pendant la stimulation. Cette valeur est donc exprimée en $\mathrm{ng} / \mathrm{ml}$. Cette réponse est nulle lors de la traite, alors qu'elle est très marquée dans les lots $A L$ et AE lorsque la vache est tétée par 3 veaux $(-0,1 ; 6,5$ et $8,5 \mathrm{ng} / \mathrm{ml}$ respectivement) (fig. 5).

Dans le lot $A L$ (existence d'un comportement maternel sélectif), la réponse de la PRL à la tétée par un seul veau est plus élevée dans le cas du veau adopté $(2,2 \mathrm{ng} / \mathrm{ml})$ que dans celui d'un veau étranger $(0,8 \mathrm{ng} / \mathrm{ml})$.

La classification des différentes réponses selon leur amplitude par rapport à la moyenne des valeurs observées avant stimulation en 4 catégories (Forte: $\mathrm{R}>200 \%$; Moyenne : $100 \%<\mathrm{R}<200 \%$; Faible : R $<100 \%$; et Nulle : $R=0$ ) (fig. 6 ) indique que, lors de la traite (lot T) ou lors de la tétée d'un veau étranger (lot $A L$ ), les réponses sont faibles ou nulles; les 4 types de réponse sont observés lors de la tétée d'un veau adopté (lot $A L$ ). Les réponses sont fortes ou moyennes lors de la tétée de 3 veaux (lots $A L$ et $A E$ ) et significativement supérieures à celles obtenues à la traite ou à la tétée d'un veau étranger. 


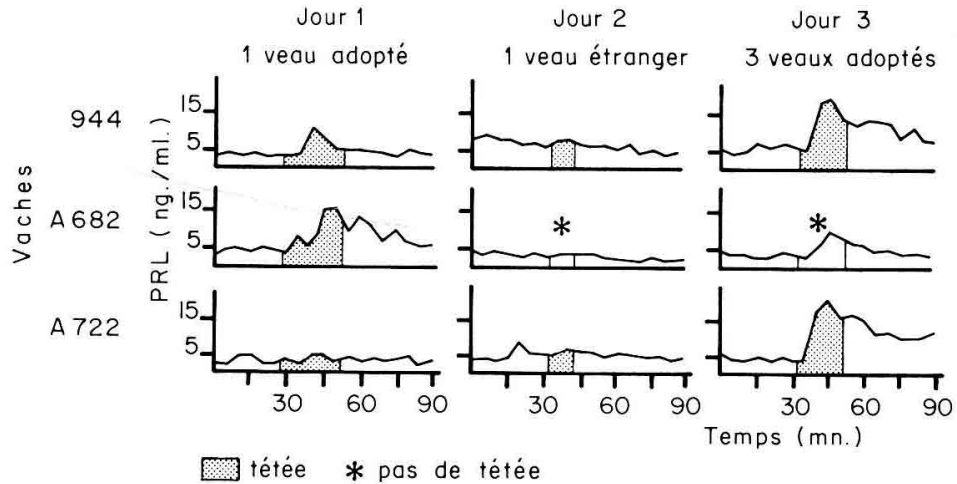

FIG. 2. - Réponse prolactinique selon la qualité de la relation mère-jeune et l'intensité de la stimulation (lot des vaches allaitantes libres - AL).

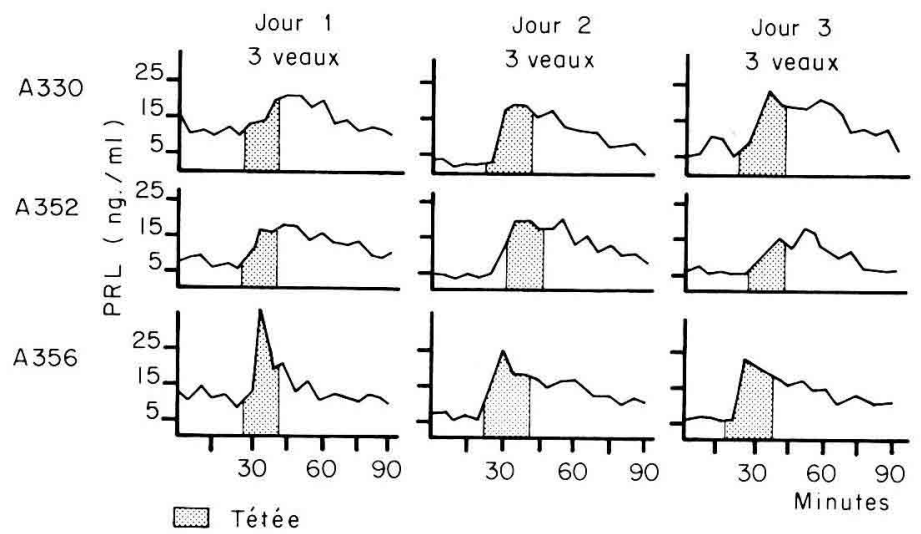

FIG. 3. - Réponse prolactinique à la tétée par troix veaux. Lot des allaitantes entravées (AE).

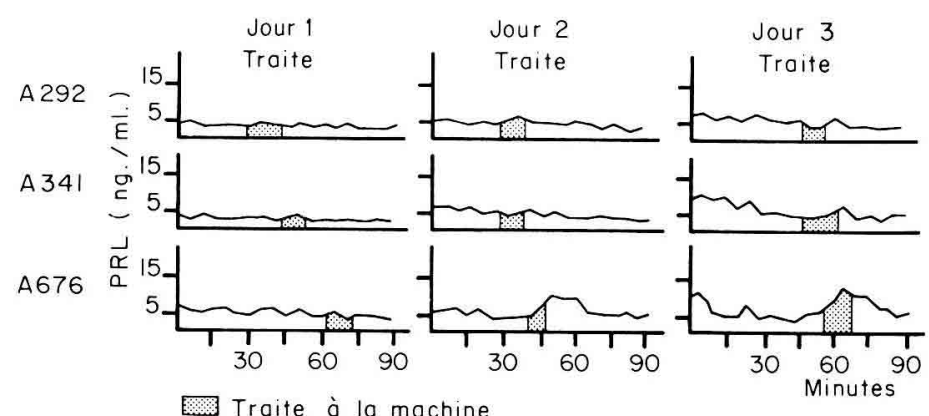

FIG. 4. - Réponse prolactinique à la traite mécanique chez les vaches du groupe traite (T). Reproduction, nutrition, développement $n^{\circ} 4 / A .-2$ 

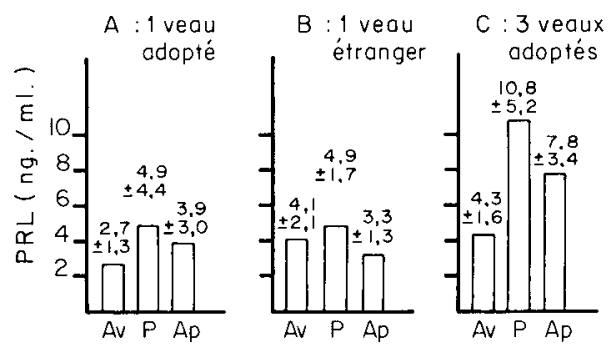
Lot $A E$ : Comportement maternel non sélectif.

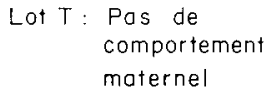
comportement maternel
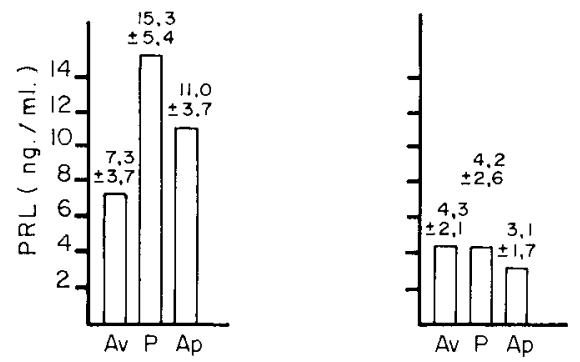

FIG. 5. - Réponse prolactinique en fonction du mode de conduite des vaches.

Av: Moyenne \pm écart-type des valeurs avant la stimulation mammaire ; $P$ : Moyenne \pm écarttype pendant la stimulation; Ap : Moyenne \pm écart-type après la stimulation.

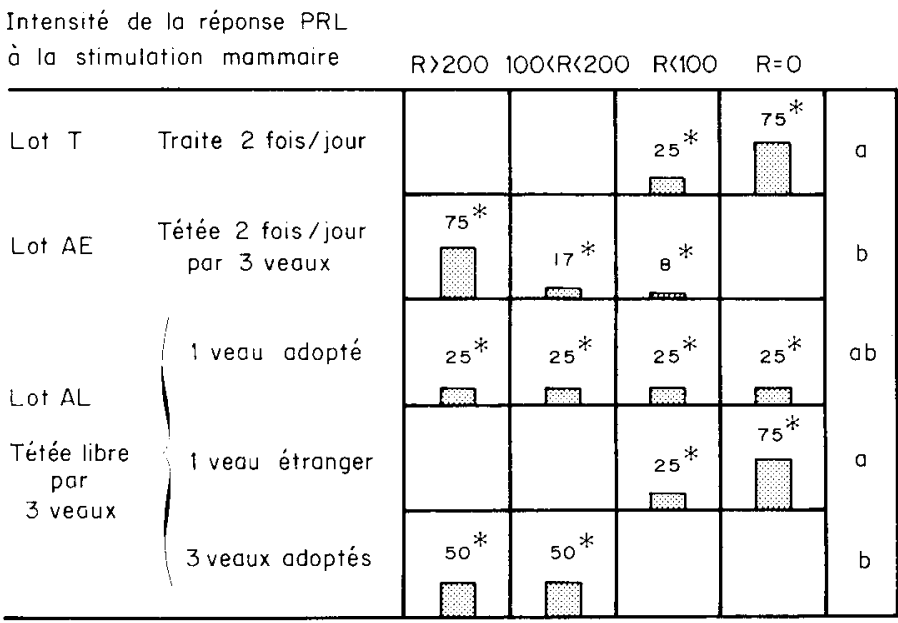

FIG. 6. - Intensité de la réponse prolactinique à la stimulation mammaire en fonction du mode de conduite (lots $A L, A E$ et $T$ ) et du nombre de veaux allaités $(A L)$.

La réponse a été exprimée selon le rapport :

$R=\frac{x \text { valeurs PRL pendant la stimulation }}{x \text { valeurs } P R L \text { avant stimulation }} \times 100$

Les groupes ayant des lettres différentes sont significativement différents $(P<0,05$, test de Kolmogorov Smirnov). 
Nous avons observé également que chez une vache du lot $A L$ dont les veaux n'ont pas voulu téter lors de la présentation (vache A682, jour 3, fig. 2), la présence des 3 veaux adoptés a suffi pour induire une réponse comprise entre 100 et $200 \%$ du niveau moyen précédant la stimulation.

En outre, nous avons constaté que dans tous les cas où une réponse PRL est obtenue, le niveau maximum de PRL est atteint avant la fin de la stimulation.

La concentration moyenne de PRL après stimulation (moyenne des valeurs après stimulation) est plus élevée qu'avant stimulation lors de la tétée par 3 veaux (lots $A L$ et $A E$ ) ou 1 veau adopté (lot $A L$ ) mais par contre est inférieure lors de la traite (lot $T$ ) ou la tétée d'un veau étranger (lot $A L$ ) (fig. 5).

\section{Discussion.}

Dans le lot des allaitantes libres, le nombre moyen des tétées par jour et par veau $(3,6 \pm 1,5)$ a été similaire à celui observé par Le Neindre et Garel (1977). Les veaux adoptés ont tété de façon préférentielle en position parallèle inversée sous leur nourrice et ils ont été plus fréquemment léchés que les veaux étrangers. Ces vaches en stabulation ont donc eu un comportement analogue à des animaux au pâturage. En revanche, contrairement à ce qui est observé par ces auteurs, on n'observe aucune dichotomie claire entre les vaches qui auraient adopté leurs trois veaux et celles qui n'en auraient adopté qu'un. Cette faible sélectivité des vaches frisonnes a déjà été observée par Poindron et Le Neindre (1979).

Les activités des animaux des deux autres groupes ont été très différentes de celles observées dans le lot AL.

Les vaches qui allaitent entravées deux fois par jour (lot $A E$ ) acceptent les veaux à la tétée et les lèchent, même lorsqu'elles sont en liberté. Ces vaches manifestent donc clairement un comportement maternel. Cependant, à l'opposé des allaitantes libres, aucune relation préférentielle n'existe (absence de sélectivité des vaches envers les veaux). Ceci suggère par conséquent que la période de contact post-partum (18 h environ) des vaches avec 3 veaux n'a pas permis l'établissement de relations sélectives durables.

Enfin, la plupart des vaches traites ne se sont pas laissées téter. Le maintien de la motivation maternelle au-delà de la période immédiatement post-partum chez la vache ne dépend donc pas uniquement de l'existence de la lactation et de la répétition de la stimulation de la mamelle. La présence du jeune dans les heures qui suivent sa naissance apparaît indispensable. Ceci rejoint les conclusions avancées par d'autres auteurs (Le Neindre et Garel, 1976 ; Hudson et Mullord, 1977) qui ont montré l'existence d'une période sensible pour la mise en place d'un comportement maternel durable chez la vache après la parturition. Cependant, certaines vaches traites se sont laissées téter très fréquemment, ce qui correspond soit à l'existence d'un comportement maternel maintenu malgré l'absence de veau, soit à une absence totale de sélectivité vis-à-vis de la nature de la stimulation mammaire.

La comparaison des réponses prolactiniques observées dans les 3 lots à la suite de la stimulation mammaire montre que la décharge observée chez les 
vaches allaitant des veaux (lots $A L$ et $A E$ ) est beaucoup plus marquée que chez les vaches traites. Ces différences peuvent provenir de deux facteurs indépendants. D'une part les réponses prolactiniques plus marquées chez les vaches maternelles (groupes $A L$ et $A E$ ) suggèrent que l'existence d'une motivation maternelle peut influencer la décharge de PRL. Ces résultats sont en accord avec ceux de Fulkerson, Hooley et Findlay (1978) bien que d'après ces auteurs l'augmentation du taux de prolactine soit sensible aussi bien chez les vaches traites que chez les vaches allaitantes. L'examen détaillé des résultats obtenus chez les vaches allaitantes libres tend à confirmer l'hypothèse selon laquelle les relations mèrejeune influencent la décharge de PRL. Ainsi, la tétée par un veau étranger lqui est en temps normal refusé par la mère) n'a provoqué dans aucun cas une décharge notable de PRL (cf. fig. 5).

Malgré le petit nombre d'animaux impliqués, ceci suggère que la sélectivité de la relation mère-jeune peut moduler la réponse PRL à la stimulation mammaire. L'absence de réponse à la suite de la tétée par un veau étranger correspond d'autant plus probablement à une inhibition que l'on sait par ailleurs qu'une stimulation même minime (lavage et massage du pis) peut conduire à une libération de prolactine (Johke, 1970 ; Karg et Schams, 1974 ; Reinhardt et Schams, 1975). De plus, on peut voir dans la fig. 2 qu'une vache a présenté une décharge de PRL très claire en présence de ses trois veaux bien que ceux-ci n'aient pas tété, alors qu'elle n'avait pas réagi lors de la présentation d'un veau étranger (femelle A682). Ce résultat est à rapprocher de ceux de Grosvenor et Mena (1971) qui indiquent que la seule présence des ratons peut provoquer une décharge de prolactine chez la rate. En revanche, Gordon et al. (1979) rapportent une inhibition de la réponse prolactinique lors de la traite mécanique chez des vaches traites en présence de leur veau. Toutefois, les conditions expérimentales et le stade de lactation auxquels ces études ont été réalisées diffèrent des nôtres et peuvent expliquer ces différences.

Le deuxième facteur pouvant expliquer la faible décharge de PRL observée chez les vaches traites tient à la nature de la stimulation mammaire (jeune dans les lots $A L$ et $A E$, machine à traire dans le lot $T$ ). Ce facteur ne saurait être exclu dans la mesure où les deux types de stimulation n'ont pas été comparés au sein du lot $T$. Un argument en faveur d'une telle hypothèse provient de la différence marquée de production laitière, supérieure de $50 \%$ chez les vaches allaitantes (groupes $\mathrm{AL}$ et $\mathrm{AE}$ ) par rapport à celle des vaches traites (lot $\mathrm{T}$ - Perez et al., 1983). Une différence aussi élevée pourrait suggérer une mauvaise efficacité de la machine à traire utilisée dans notre expérience. D'un autre côté il est peu probable que la complète absence de réponse PRL observée chez les vaches traites puisse s'expliquer uniquement par un défaut de la machine. On sait en effet qu'une stimulation telle que le lavage du pis, ou son massage suffisent pour obtenir une réponse PRL (Johke, 1970 ; Karg et Schams, 1974 ; Reinhardt et Schams, 1975).

Nous avons aussi observé que la quantité de PRL libérée est en relation avec l'intensité de la stimulation à la tétée (nombre de trayons stimulés); ainsi, elle est plus élevée quand la vache est tétée par 3 veaux que par un seul. Ces résultats sont en accord avec ceux de Karg et Schams (1974). Par contre, la durée de la 
décharge ne semble pas dépendre de la durée de la stimulation. En effet, dans la majorité des cas (14/17), le niveau de PRL commence à descendre avant la fin de la stimulation, comme cela avait déjà été constaté par Tucker (1971) et Gordon et al. (1979), à l'occasion de la traite à la machine. La moyenne des valeurs après stimulation très élevée dans le cas de la tétée par 3 veaux (lots AL et AS) correspond à des cas où la réponse à la stimulation est aussi la plus forte. En d'autres termes plus la réponse à la stimulation est forte, plus l'intervalle de temps nécessaire pour atteindre le niveau de base est long.

En conclusion, ces premiers résultats confirment que la libération de PRL peut être influencée par l'intensité de la stimulation mammaire et indiquent que le mode de conduite des mères et les facteurs comportementaux qui y sont associés peuvent également jouer sur la décharge prolactinique.

Reçu en novembre 1983. Accepté en mars 1985.

\section{Références}

FELL L. R., BECK C., BLOCKEY M. A. de B., BROWN J. M., CATT K. J., CUMMING I. A., GODING J. R., 1971. Prolactin in the dairy cow during suckling and machine milking. J. Reprod. Fert., 24, 144-145.

FELL L. R., BECK Ch., BROWN J., CATT K. J., CUMMING I. A., GODING J. R., 1972. Solid phase radioimmunoassay of ovine prolactin in antibody-coated tubes. Prolactin secretion during estradiol treatment at parturition and during milking. Endocrinology, 91, 1329-1337.

FRASER A. F., 1968. Reproductive behaviour in ungulates. Acad. Press, London, 202 p.

FULKERSON W. J., HOOLEY R. D., FINDLAY J. K., 1978. Improvement of milk production of first calf heifers by multiple suckling. Aust. $J$. agric. Res., 29, 351-357.

GORDON T., DOODMAN H., TUCKER A., CONVEY E. M., 1979. Presence of the calf affects secretion of prolactin in cows (40565). Proc. Soc, exper. Biol. Med. 161, 421-424.

GROSVENOR C. E., MENA F., 1971. Effect of suckling upon the secretion and release of prolactin from the pituitary of the lactating rat. IX Biennial Symposium on Animal Reproduction. $J$. anim. Sci., 32, 115-135.

BRYANT G. D., GREENWOOD F. C., LINZELL J. L., 1968. Plasma prolactin levels in the goat : physiological and experimental modification. J. Endocr., 40, IV.

HART I. C., 1974. The relationship between lactation and the release of prolactin and growth hormone in the goat. J. Reprod. Fert., 39, 485-499.

HUDSON S. J., MULLORD M. M., 1977. Investigations of maternal bonding in dairy cattle. Appl. Anim. Ethol., 3, 271-276.

JOHKE Tetsu, 1969. Prolactin release in response to milking stimulus in the cow and goat estimated by radioimmunoassay. Endocr. japan, 16, 179-185.

JOHKE Tetsu, 1970. Factors affecting the plasma prolactin level in the cow and the goat as determined by radioimmunoassay. Endocr. japan, 17, 393-401.

KANN G., 1971. Dosage radioimmunologique de la prolactine plasmatique chez les ovins. C.R. Acad. Sci., Paris, sér. D., 272, 2808-2811.

KANN G., CARPENTIER M. C., FEVRE J., MARTINET J., MAUBON M., MEUSNIER C., PALY J., VERMEIRE N., 1978. Lactation and prolactin in sheep, role of prolactin in initiation of milk secretion, 201-212. In C. ROBYN and M. HARTER. Progress in prolactin physiology and pathology. Elsevier/North Holland biomed. Press.

KARG H., SCHAMS D., 1974. Prolactin release in cattle. J. Reprod. Fert., 39, 463-472. 
LE NEINDRE P., GAREL J. P., 1976. Existence d'une période sensible pour l'établissement du comportement maternel de la vache après la mise bas. Biol. Behav., 1, 217-221.

LE NEINDRE P., GAREL J. P., 1977. Etude des relations mère-jeune chez les bovins domestiques : comparaison des liaisons existant entre la mère et des veaux légitimes ou adoptés. Biol. Behav., 2, 39-49.

PEREZ O., JIMENEZ DE PEREZ N., LE NEINDRE P., COCHAUD J., 1983. Production laitière des vaches Pie Noires traites ou allaitant 3 veaux. Ann. Zoot., 32, 475-482.

POINDRON P., LE NEINDRE P., 1975. Comparaison des relations mère-jeune observées lors de la tétée chez la brebis (Ovis aries) et chez la vache (Bos taurus). Ann. Biol. anim. Bioch. Biophys., 15, 495-501.

PôINDRON P., LE NEINDRE P., 1979. Les relations mère-jeune chez les ruminants domestiques et leurs conséquences en production animale. Bull. tech. Départ. Génét. anim., 29-30, 33-57.

REINHARDT V., SCHAMS D., 1975. Prolactin release in dairy heifers relative to duration of teat stimulation. Neuroendocrinology, 17, 54-61.

TINDAL J. S., 1974. Hypothalamic control of secretion and release of prolactin. J. Reprod. Fert., 39, 437-461.

TUCKER-ALLEN H., 1971. Hormonal response to milking. IX Biennial Symposium on Animal Production. J. anim. Sci, 32, 137-144. 